\title{
Enneking Stage IIB
}

National Cancer Institute

\section{Source}

National Cancer Institute. Enneking Stage IIB. NCI Thesaurus. Code C146704.

A stage for malignant musculoskeletal tumors characterized by high grade (G2), intracomparmental site (T2), and no metastasis (M0). 